edge or of previous episodes, then there is no need to postulate separate short-term and long-term memory systems. However, different time courses of the task result in different time courses of the ERP trace, a result that could reflect different modes of operation (and hence, functionally different cognitive functions) of the same brain structures. Moreover, maintenance of material on a temporary basis, beyond the end of stimulus presentation, is associated with activity in the prefrontal areas, as well as in the posterior areas, which have been linked to activation of long-term memory. As Ruchkin et al. point out, the advantage of word lists over nonword lists in immediate serial- and free-recall tasks indicates an involvement of semantic information in supporting temporary memory functions. However, there is nothing in the reported ERP data that constrains the interpretation that temporary retention involves areas of the prefrontal cortex, in addition to some form of ongoing activation of the recently activated traces in long-term memory. Temporary memory appears to be associated with both anterior and posterior areas of activation. This could suggest that the prefrontal cortex is the seat of temporary memory, or that both the prefrontal and the posterior activation are required in such tasks, or that the prefrontal activation reflects the operation of some form of controlling mechanism that ensures continued maintenance of long-term memory traces. In all cases, there is a different network of activation associated with temporary memory than with long-term memory tasks, even if there is some overlap in the brain areas involved. At a conceptual level, all three of these interpretations are quite consistent with working memory holding the products of activated traces from long-term memory; they are also consistent with working memory comprising a system that is conceptually quite distinct from long-term memory.

\section{Does sustained ERP activity in posterior lexico-semantic processing areas during short-term memory tasks only reflect activated long-term memory?}

\section{Steve Majerus ${ }^{\mathrm{a}}$, Martial Van der Linden ${ }^{\mathrm{b}}$, Fabienne Collette ${ }^{\mathrm{a}}$ and Eric Salmonc \\ a Department of Cognitive Sciences, University of Liège, 4000 Liège,

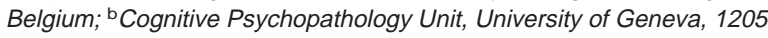 \\ Geneva, Switzerland; ' Cyclotron Research Center, University of Liège, 4000 \\ Liège, Belgium.smajerus@ulg.ac.be \\ martial.vanderlinden@pse.unige.ch f.collette@ulg.ac.be \\ eric.salmon@ulg.ac.be}

Abstract: We challenge Ruchkin et al.'s claim in reducing short-term memory (STM) to the active part of long-term memory (LTM), by showing that their data cannot rule out the possibility that activation of posterior brain regions could also reflect the contribution of a verbal STM buffer.

Ruchkin et al. argue that they provide strong evidence for a lexico-semantic contribution to verbal short-term memory (STM) tasks, by showing greater event-related potential (ERP) activity in posterior lexico-semantic processing areas (most pronounced in the vicinity of the central midline scalp) during the retention of STM lists of five words compared to STM lists of three nonwords. At an empirical level, we support Ruchkin et al.'s results, as we also observed very similar data in a recent positron emission tomography (PET) study investigating lexicality effects in STM. Collette et al. (2001) explored the activation of posterior brain areas in both a STM task (ordered recall of three words vs. three nonwords) and a control condition (repetition of one word vs. one nonword). When comparing brain regions activated for words versus nonwords in the STM condition, after accounting for brain regions already activated when contrasting words and nonwords in the control condition, we observed greater activation in two posterior brain regions, the left middle temporal gyrus (BA 21) and the left temporo-parietal junction (BA 39). Our data clearly support the idea that posterior brain areas play an active role during shortterm retention of words and further complement the data of Ruchkin et al. by providing a more precise localization of these brain regions.

However, at a theoretical level, we consider that there might be an alternative interpretation for the activation of posterior brain regions during STM processing of words than the interpretation put forward by Ruchkin et al. They consider that their data support the position that STM mainly reflects the activated state of LTM, based on similar activation of posterior brain areas thought to encode lexico-semantic language knowledge during both single-word processing and STM for words, relative to single-nonword processing and STM for nonwords. This interpretation includes the implicit assumption that the observed activation of posterior processing areas exclusively reflects the neural substrate of lexico-semantic representations encoded in LTM. However, we think that the results cannot exclude the possibility that the posterior brain areas activated in that study could also reflect the contribution of a STM buffer in addition to activation of lexico-semantic knowledge in LTM. First, the posterior regions encoding lexico-semantic knowledge and those having a buffer function, although different, could be spatially very close and thus difficult to distinguish by ERP techniques, which have a relatively poor spatial resolution. Second, the authors compared recall of five words versus three nonwords in order to achieve a similar level of STM performance for words and nonwords. However, a similar level of performance does not guarantee that the requirements of STM storage capacities are the same in both tasks; it is still possible that STM load was greater in the word than in the nonword condition, especially as the words (five items) had to be maintained longer in STM than the nonwords (three items) before recall. By extension, this implies that the greater ERP activation observed in posterior brain regions during STM for words relative to nonwords could also reflect the activation of a STM buffer, and not only activation of lexico-semantic knowledge as proposed by the authors. Third, we recently showed more directly that posterior brain regions could have a specific STM buffer function for verbal information, by studying brain activation using PET imaging for verbal STM performance in three patients that had recovered from LandauKleffner syndrome, a rare epileptic childhood aphasia characterized by persistent verbal STM impairments (Majerus et al. 2003a). The patients were presented lists of four words for immediate serial recall and a control condition (repetition of one word); there was also a control group of 14 healthy young adults. Two of the patients showed reduced activation in left and right posterior superior temporo-parietal areas during the STM condition compared to the control condition, and they presented, at the same time, impaired performance in the STM condition. The third patient showed increased activation in the right posterior superior temporo-parietal area in the STM condition, while presenting, at the same time, relatively normal STM performance. These results suggest that activation of the posterior temporo-parietal area determines very directly the level of performance observed in the STM condition. Furthermore, as there were no differences in behavioural and imaging results between control subjects and the patients for repetition of single words (control condition), which required the same amount of activation of lexico-semantic representations as the STM condition (repetition of word lists), impaired lexico-semantic activation is not likely to account for the results observed in the STM condition. Finally, in a neuropsychological study with the same patients, we explored more directly the relationship between language-processing impairments and verbal STM performance (Majerus et al. 2003b); we showed that all three patients showed no major impairment at the level of lexicosemantic representations, using both standard vocabulary tests as well as more sensitive experimental tasks measuring speed of access to lexico-semantic representations. Furthermore, although two of the patients showed some residual deficits in phonological 
processing, the severity of these phonological processing impairments was not related to the severity of the verbal STM impairments for both phonological and lexico-semantic information, thereby showing dissociations between STM storage capacity and the integrity of language representations. Altogether, our results suggest that posterior temporo-parietal areas are specifically related to verbal STM processing and could play the role of a verbal STM buffer, while the results cannot be easily explained by reducing the involvement of these areas during verbal STM tasks simply to the activation of lexico-semantic knowledge. Similar claims have been made by Collette et al. (2001), Giraud and Price (2001), Grasby et al. (1993), and Wise et al. (2001).

To conclude, we argue that the data reported by Ruchkin et al., although consistent with their view of STM as an activated state of language representations stored in posterior brain regions, do not rule out the existence of a verbal STM buffer, which might also be sustained by posterior brain regions very close to those encoding LTM lexico-semantic representations.

\section{ACKNOWLEDGMENTS}

Steve Majerus is a Postdoctoral Researcher and Fabienne Collette is a Senior Research Associate; both are at the Fonds National de la Recherche Scientifique (Belgium).

\section{Cognitive neuropsychological evidence for common processes underlying generation and storage of language representations}

\section{Nadine Martin}

Department of Communication Sciences, Temple University, Philadelphia, PA 19122.nmartin@temple.edu

\begin{abstract}
Ruchkin et al. offer a compelling case for a model of short-term storage without a separate buffer. Here, I discuss some cognitive neuropsychological data that have been offered in support of and against their model. Additionally, I discuss briefly some new directions in cognitive neuropsychological research that bear on the role of attention in Ruchkin et al.'s model.
\end{abstract}

Speaking and comprehending language take place over time. It is this temporal component of language processing that marks the intersection of memory and language systems. Research efforts have devoted much attention to revealing the organization of each system. The target article by Ruchkin et al. reflects more recent efforts to understand how these two systems are related cognitively and neurophysiologically. The authors provide important evidence supporting a model that attributes temporary storage of language representations to short-term activation of long-term representations without the necessity of a buffer to house the contents of short-term memory (STM).

Cognitive neuropsychological data have been an important source of arguments both for and against the separate buffer model. Evidence supporting an independent buffer comes mainly from case studies of individuals with impaired processing of multiple words in the context of good processing of single words, forming what appears to be a dissociation between word processing and storage systems (Shallice 1988; Vallar \& Shallice 1990). The logic of this argument rests on the counter-intuitive assumption that a buffer stores only multiple word sequences. The postulation of a separate buffer is independent of assumptions about the contents of the buffer. Independent buffers are incorporated into models that assume only phonological representations in STM (Baddeley 1986), as well as those that assume short-term stores for all "levels" of linguistic representation (R. Martin \& Lesch 1996).

Neuropsychological evidence also supports the model advanced by Ruchkin et al., in which linguistic representations activated during single- or multiple-word processing are maintained by virtue of the same activation processes that enable activation and retrieval of the representations in the first place. My colleagues and I (Martin et al. 2000) have framed this relationship of language and short-term memory within an interactive activation model of word processing (Dell \& O’Seaghdha 1992), with additional components that encode serial order of multiple word sequences (Gupta 1996). In this model, maintenance of activated representations is achieved by two parameters of spreading activation, its strength and its duration. Impairments of single- and multiple-word processing are viewed as disturbances of either or both of these parameters and are assumed to lie on a continuum of severity. Milder activation impairments allow for maintenance and successful processing of single words, but not multiple words, giving the appearance of a selective deficit of verbal short-term memory. More severe activation impairments lead to difficulty in maintaining activation of linguistic representations, even during performance of single-word language tasks, leading to what are typically described as aphasic deficits. In addition to severity of impairment, the ability to maintain activation of linguistic representations in the short term is dependent on the locus of impairment (e.g., semantic, phonological) and task demands.

This model has received empirical support, in part, from studies revealing systematic associations between linguistic and STM impairments (N. Martin \& Saffran 1997; R. Martin et al. 1994). Numerous studies indicate that span performance in normal and impaired subjects is sensitive to linguistic variables, including phonological (e.g., Conrad 1964), lexical (e.g., Berndt \& Mitchum 1990; Saffran \& N. Martin 1990), semantic (e.g., R. Martin et al. 1994; Shulman 1971), and conceptual (e.g., Potter 1993; Saffran \& N. Martin 1999) aspects of words. Additionally, in aphasia, span varies based on the nature of the task used to assess span, in conjunction with the nature of the language impairment (N. Martin 1999; N. Martin \& Ayala, submitted). Finally, although long-term learning of language is unaffected by impairments affecting storage of phonological representations (e.g., Vallar \& Baddeley 1984), it is disrupted in the case of semantic and semantic-STM impairments (N. Martin \& Saffran 1990; 1999; Freedman \& R. Martin 2001).

Demonstration of these associations is necessary, but not sufficient, evidence for a model that presupposes common processes underlying generation and temporary storage of language representations. Two additional avenues of inquiry provide more definitive support. First, studies of corecovery of word-processing and verbal-STM impairments (N. Martin et al. 1994; 1996) indicate that increased capacity to temporarily store words coincides with improved ability to retrieve words without error. Second, N. Martin \& Gupta (2004) demonstrated a severity continuum between impairments of single-word and multiple-word processing, showing that performances on three measures of word processing (auditory lexical decision, picture naming, and word comprehension) correlated with auditory-verbal spans in a group of 50 individuals with aphasia, ranging from mild to severe. These recovery and severity continuum data are consistent with the model advanced by Ruchkin et al. and offer an alternative to the separate buffer model as an account of good single-word processing coupled with impaired multiple-word processing.

Ruchkin et al. propose that prefrontal attentional systems serve to coordinate the short-term activation of posteriorly represented linguistic representations. The role of attentional systems in the breakdown of language and STM functions in aphasia is a relatively recent area of investigation. A study by Hamilton and R. Martin (2002) provides relevant evidence. They demonstrated the presence of inhibition and proactive interference effects in span performance of an individual with aphasia-related semantic impairment, secondary to a lesion affecting the inferior frontal cortex. This is a promising line of research and illustrates, once again, that behavioural studies of impaired cognitive processes, such as language, STM, and attention, can provide important insights into the coordination of multiple systems that bear on performance of verbal tasks. 